\title{
Correction to: Exoskeleton with Soft Actuation and Haptic Interface
}

Ivanka Veneva, Dimitar Chakarov, Mihail Tsveov, and Pavel Venev

\section{Correction to:}

Chapter "Exoskeleton with Soft Actuation and Haptic

Interface" in: M. C. Carrozza et al. (Eds.): Wearable Robotics:

Challenges and Trends, BIOSYSROB 22,

https://doi.org/10.1007/978-3-030-01887-0_79

In the original version of the book, the following belated corrections have been incorporated: The author name "Michail Tsveov" has been changed to "Mihail Tsveov" in the Frontmatter, Backmatter and in Chapter. 